\title{
Sensitivity of Signal-to-Noise Ratio to the Layer Profile and Crystal Quality of SiGe/Si Multilayers
}

\author{
H. H. Radamson, ${ }^{\text {a,z }}$ M. Moeen, ${ }^{a}$ A. Abedin, ${ }^{\text {a,* }}$ A. Hojabri, ${ }^{a}$ A. Salemi, ${ }^{a}$ and M. Kolahdouz ${ }^{b}$ \\ ${ }^{a}$ KTH Royal Institute of Technology, School of Information and Communication Technology, 16640 Kista, Sweden \\ ${ }^{b}$ School of Electrical and Computer Engineering, University of Tehran,Tehran, Iran
}

\begin{abstract}
This study presents signal-to-noise ratio (SNR) measurements of single crystalline dots or layers of SiGe/Si in multilayer structures in terms of Ge content, interfacial and layer quality. All multilayers were processed in form of mesas and the noise behavior of electrical signal was investigated by comparing the power spectral density curves and $\mathrm{K}_{1 / \mathrm{f}}$ values. The $\mathrm{SiGe} / \mathrm{Si}$ multilayer structures were also characterized by the conventional material analysis tools and the results were compared to the noise measurements. The quality of SiGe/Si interface or SiGe layer was monitored by intentional exposure to oxygen in range of 2-1600 nTorr either during or prior to SiGe growth. The results demonstrated that SNR was sensitive to the interfacial and layer quality, and the Ge content in a multilayer structure. The noise level became very high when the strain fluctuated within SiGe layer and this occurred for SiGe with high Ge content or SiGe dots.
\end{abstract}

(C) 2016 The Electrochemical Society. [DOI: 10.1149/2.0261604jss] All rights reserved.

Manuscript submitted December 8, 2015; revised manuscript received February 15, 2016. Published February 24, 2016. This paper

is part of the JSS Focus Issue on Defect Characterization in Semiconductor Materials and Devices.

SiGe alloys have been widely integrated in large variety of devices such as CMOS for strain engineering, ${ }^{1-3}$ bipolar transistors, ${ }^{4,5}$ photonic detectors, ${ }^{6}$ bolometers, ${ }^{7-9}$ quantum cascade lasers ${ }^{10}$ and bio- or gas-sensors. ${ }^{11-13}$ For these applications, SiGe material is grown at low temperatures $\left(350-650^{\circ} \mathrm{C}\right)$ where the quality of layers and interfaces plays a critical role in device characteristics. Since high strain amount is often sought then layers with high Ge content have to be grown below the critical thickness in the metastable region. One problem with epi-layers grown at low temperatures is oxygen and moisture contamination which is rooted from epitaxy environment and the purity of the reactant gases. ${ }^{14,15}$

An important criterion in all types of devices is the signal-tonoise ratio (SNR) which indicates the quality of the electrical signal. Noise measurement is a sensitive method which can provide accurate information about any minor defects in the crystal.

In this article, $\mathrm{SiGe}$ material is grown at low temperatures and integrated in a multilayer structure with $\mathrm{Si}$ barriers (e.g. SiGe/Si) where the number of periodicity, layer and interface quality were studied by noise measurements. The $\mathrm{SiGe}$ material was either in form of two-dimensional layer or dots. Furthermore, the integrity of epitaxial growth at low temperatures in terms of oxygen contamination was presented. A discussion of how sensitive the conventional characterization tools are compared to noise measurements for detection of defects has also been included.

\section{Experimental}

The $\mathrm{SiGe} / \mathrm{Si}$ multilayer structures were deposited on $\mathrm{Si}(100)$ substrates in temperature range of $350-650^{\circ} \mathrm{C}$ at 20 torr using reduced pressure chemical vapor deposition (RPCVD). The reactant gases for SiGe growth were $\mathrm{GeH}_{4}$ or $\mathrm{Ge}_{2} \mathrm{H}_{6}$ and $\mathrm{SiH}_{4}$ or $\mathrm{Si}_{2} \mathrm{H}_{6}$ as $\mathrm{Ge}$ and $\mathrm{Si}$ sources, respectively. All SiGe layers were grown below the critical thickness according to the published data. ${ }^{15-17}$ The quality of epi-layers and $\mathrm{SiGe} / \mathrm{Si}$ interface could be monitored by exposing to oxygen during epitaxy or at the interfaces. For these experiments, the oxygen partial pressure was in range of $2-1600$ nTorr. In order to determine the flux and absorption of oxygen in the reactor a residual gas analyzer (RGA) was connected in the exhaust gas line.

Secondary ion mass spectrometry (SIMS) was applied to determine the level of oxygen in the grown layers.

The samples consisted of either SiGe multi-quantum wells or dots (MQWs or MQDs) in a stack with $52 \mathrm{~nm}$ Si barrier layers. The thick- ness of epi-layers was $10 \mathrm{~nm}$ with Ge contents in range of $21-47 \%$ whereas the Ge dots had diameters within $3-50 \mathrm{~nm}$ and a surface density of $2 \times 10^{10} \mathrm{~cm}^{-2}$. The composition and thickness of the $\mathrm{SiGe}$ layers were obtained from analysis of high-resolution X-ray diffraction rocking curves. These curves were simulated by using Takagi-equations in order to have precise information about the structure profile as shown in Fig. 1. ${ }^{18}$ High-resolution reciprocal lattice mappings (HRRLM) were also performed to determine the misfit parameters and relaxation amount. The multilayers were sandwiched between highly B-doped layers with concentration of $5 \times 10^{19} \mathrm{~cm}^{-3}$ for contacting. In order to avoid auto-doping or out-diffusion of boron into the $\mathrm{SiGe} / \mathrm{Si}$ layers, $\mathrm{Si}$ spacers of $300 \mathrm{~nm}$ were grown on the top and bottom of multilayer structures as shown in Fig. 2. The mesas were formed by dry etching with areas of $70 \times 70,100 \times 100,140 \times$ 140 , and $200 \times 200 \mu \mathrm{m}^{2}$.

These mesas were passivated by $100 \mathrm{~nm} \mathrm{Si}$ oxide. The contact areas were opened and $\mathrm{Ni}$ silicide was formed at $450^{\circ} \mathrm{C}$ prior to metallization of TiW/Al. The purpose of Ni silicide layer is to reduce the contact resistance which may act as a noise source in the signal analysis.

The silicide process created mostly NiSi phase according to X-ray analysis. ${ }^{19}$ Higher annealing temperatures were avoided in order to minimize the intermixing of $\mathrm{Si}$ into Ge dots. The silicide layers were

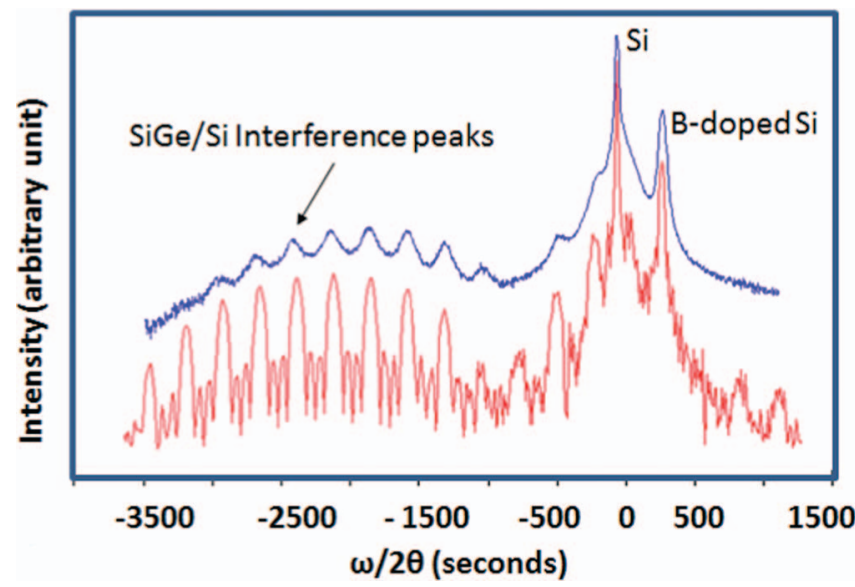

Figure 1. X-ray rocking curve of $\mathrm{Si}_{0.72} \mathrm{Ge}_{0.28} / \mathrm{Si}$ multilayer structures with four periods (in blue color) and simulated curve (in red color). $\omega$ and $2 \theta$ stand for incident and diffracted angles, respectively. 


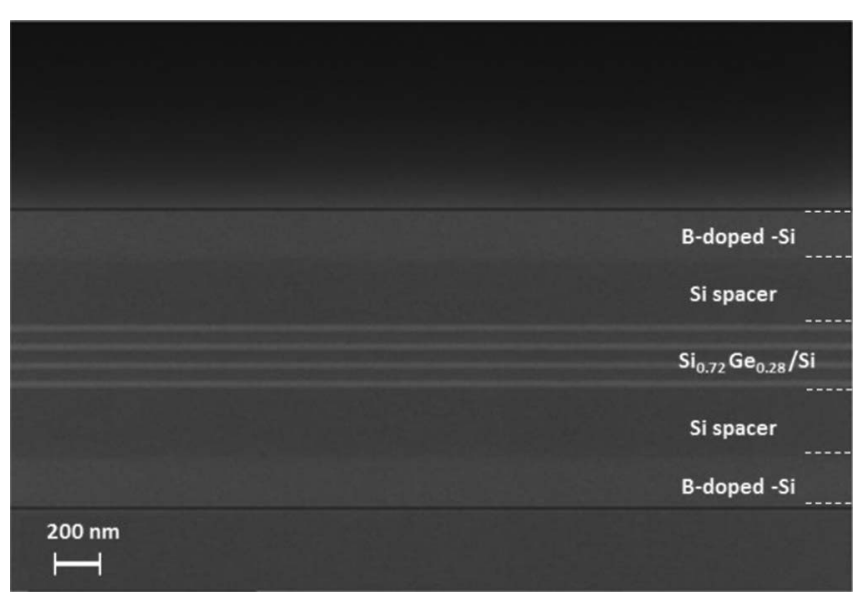

Figure 2. High-resolution scanning electron microscope (HRSEM) crosssectional image of SiGe/Si multilayer structure in Fig. 1.

located far from $\mathrm{SiGe}$ layers to ensure no strain relaxation occurs in $\mathrm{SiGe} / \mathrm{Si} \mathrm{MQWs}{ }^{20}$

The quality of SiGe layers was examined by introducing oxygen (2-1600 nTorr) at the interfaces of $\mathrm{SiGe} / \mathrm{Si}$. These samples were prepared to study the effect of interface quality on the electrical transport in the mesas. Figs. 3a-3c summarize the process steps and the designed structures in this study.

The noise measurements were performed by using a Cascade 11000 shielded probe station which is equipped with a battery powered resistive network together with a low-noise voltage pre-amplifier and a HP49810 vector spectrum analyzer. An appropriate applied voltage $\left(\mathrm{V}_{\text {bias }}\right)$ for the mesas could be in the range of $0.5 \mathrm{~V}$ to $5 \mathrm{~V}$ and in this
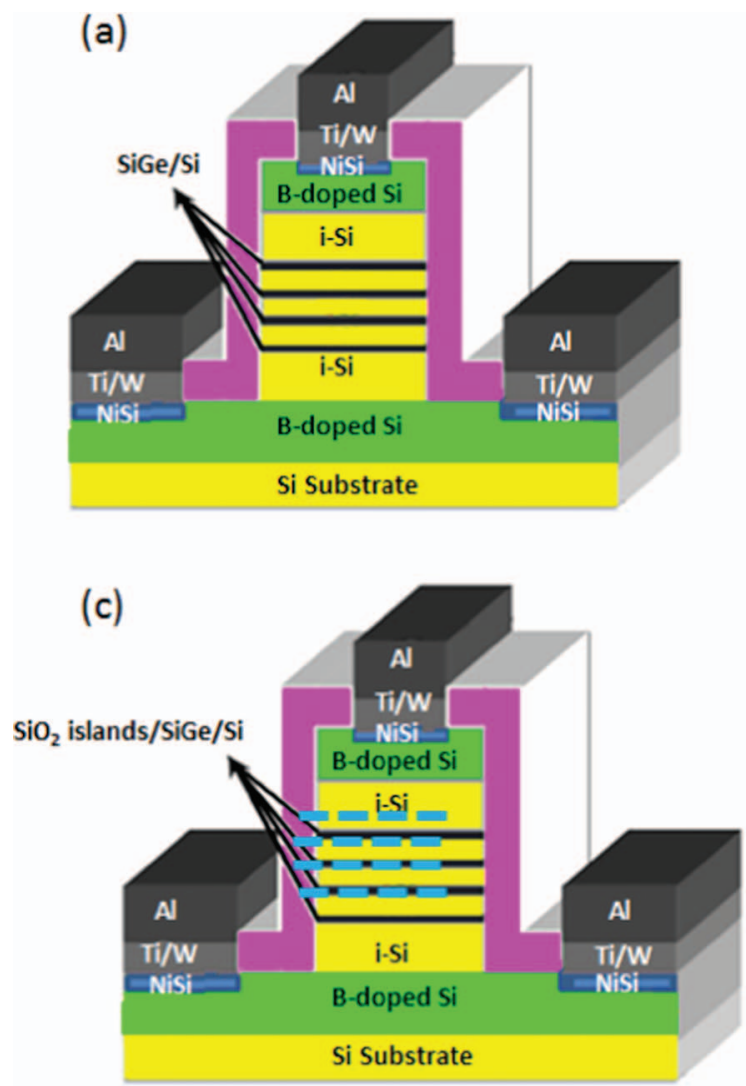

study, $\mathrm{V}_{\text {bias }}=1 \mathrm{~V}$ was chosen in order to avoid any undesired heat generation in the mesas. The power spectral density (PSD) of noise voltage was measured and compared for different types of $\mathrm{SiGe} / \mathrm{Si}$ samples.

In general, $1 / f$ noise is caused by interactions of carriers with impurities, imperfections in the crystal and surface states where a trapping/detrapping phenomenon occurs. In semiconductors, $1 / f$ noise is usually expressed by noise constant according to:

$$
\mathrm{K}_{1 / f}=\alpha_{\mathrm{H}} / \mathrm{N}
$$

where $\mathrm{N}$ stands for the total number of free charges and $\alpha_{H}$ is the Hooge's constant. ${ }^{21}$

The current and voltage noise $\left(\mathrm{S}_{\mathrm{I}}\right.$ and $\mathrm{S}_{\mathrm{V}}$, respectively) in $\mathrm{SiGe} / \mathrm{Si}$ stack can be formulated as following: ${ }^{22}$

$$
\begin{aligned}
S_{I} & =\frac{K_{1 / f} I_{\text {sample }}^{2}}{f^{\gamma}}=\frac{K_{1 / f}\left(V_{\text {bias }} / R_{\text {sample }}\right)^{2}}{f^{\gamma}} \\
& \Rightarrow S_{V}=\left(R_{\text {sample }}\right)^{2} S_{I}=\frac{K_{1 / f} V_{\text {bias }}{ }^{2}}{f^{\gamma}}
\end{aligned}
$$

where $\mathrm{I}_{\text {sample }}$ and $\mathrm{R}_{\text {sample }}$ refer to the current and resistance of the device during the measurement, respectively. In these equations, the power of frequency, $\gamma$ is nearly 1 . If the performance of $1 / f$ noise deviates from value 1 , this is mostly rooted from generation-recombination (GR) noise caused by defects in the $\mathrm{SiGe/Si} \mathrm{multilayer} \mathrm{structure} \mathrm{or}$ at the interface of the oxide-passivated surface of the mesa body. The $\mathrm{K}_{1 / \mathrm{f}}$ is acquired by the slope of the decaying part of the PSD curve.

\section{Results and Discussion}

SiGe material is usually deposited at low temperatures in order to have highly strained layers with precisely controlled thicknesses.

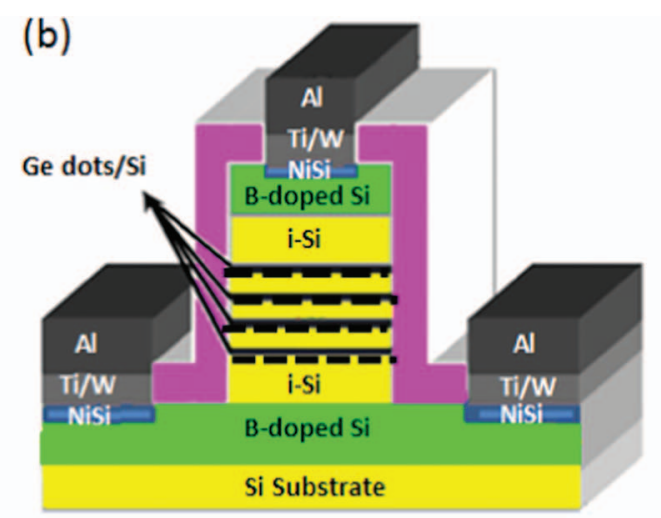

Epitaxy of multilayer of $\mathrm{SiGe} / \mathrm{Si}$ or $\mathrm{Ge}$ dots/Si

- $\mathrm{SiO}_{2}$ deposition and hard mask formation

- Dry etch and formation of mesas

- $\mathrm{SiO}_{2}$ passivation

- Dry etch to stripe oxide contact openings

- Ni Salicide formation

- Metallization

- Metal etch and formation of contacts and pads

- Forming gas annealing

Figure 3. $(a-c)$ The listed process steps for samples which are manufactured for electrical characterization in this study together with schematic pictures of these samples: a) $\mathrm{SiGe} / \mathrm{Si}$ multilayers, b) same as a) but with $\mathrm{SiO}_{2}$ islands at interfaces of $\mathrm{SiGe} / \mathrm{Si}$ and $\mathrm{Ge}$ dots/Si multilayers. 

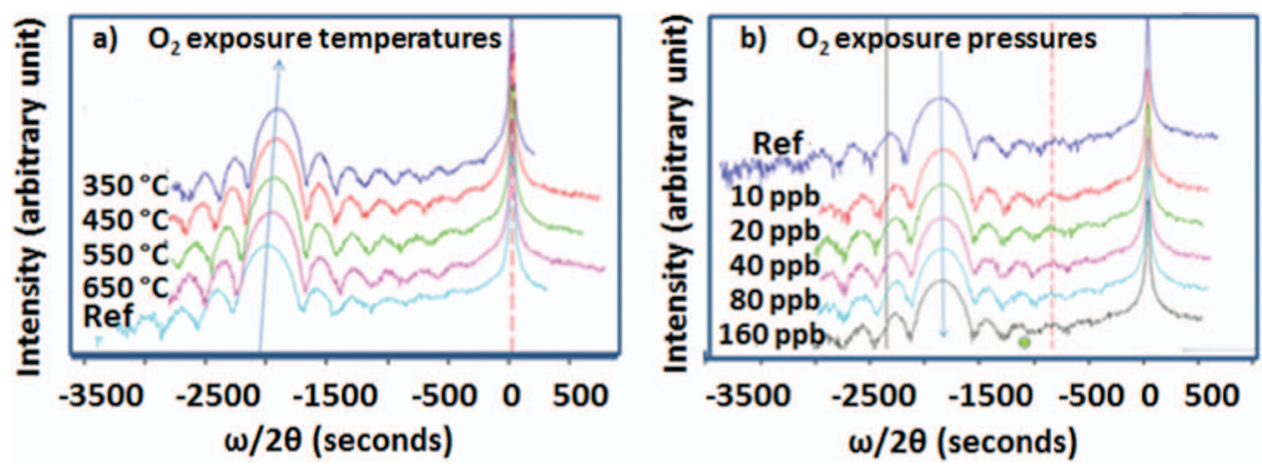

Figure 4. (a) X-ray $\mathrm{RCs}$ from $\mathrm{Si}_{0.79} \mathrm{Ge}_{0.21}$ layers grown at low growth temperatures with $10 \mathrm{nTorr}$ oxygen partial pressure and (b) $\mathrm{Si}_{0.79} \mathrm{Ge}_{0.21}$ layers grown with different oxygen exposed partial pressures.

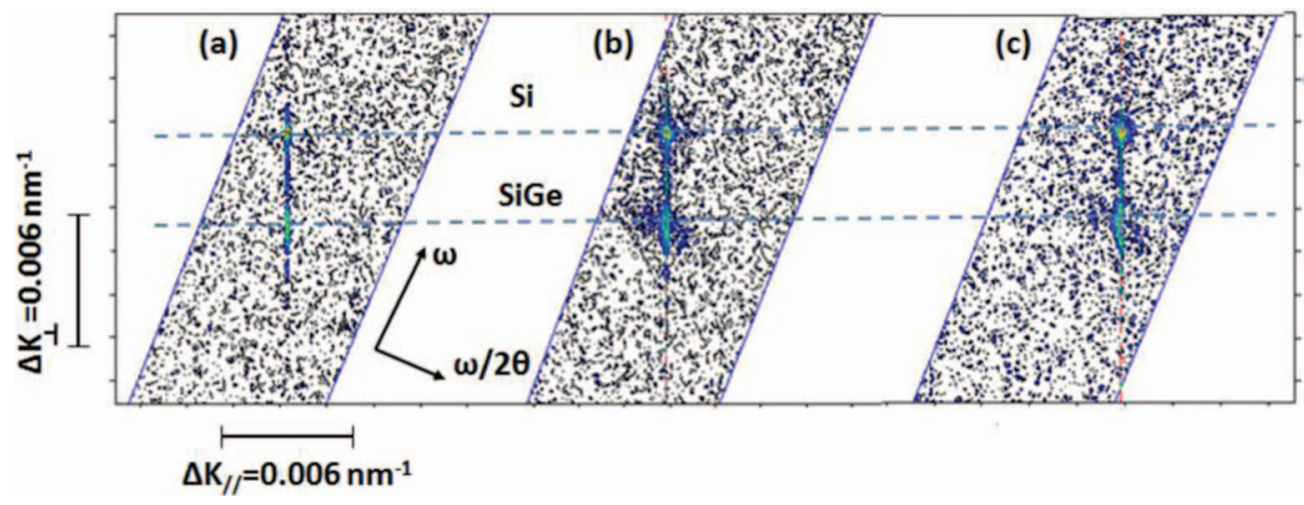

Figure 5. (a-c) HRRLMs around (113) reflection from $\mathrm{Si}_{0.79} \mathrm{Ge}_{0.21}$ layers grown with $10 \mathrm{nTorr}$ oxygen at (a) $550^{\circ} \mathrm{C}$, (b) $600^{\circ} \mathrm{C}$ and (c) $650^{\circ} \mathrm{C}$. The color contours present different X-ray intensities measured by detector.

At low growth temperatures, the quality of process gases and epitaxy environment in terms of oxygen or moisture level is an issue. ${ }^{23-25}$ The source of this problem could be related to purity of the reactant gases (although the highest purity gases are used) and the presence of oxygen and moisture in the load-locks when the samples are loaded (usually no baking is performed). Although the background contamination level is minor and it could be in range of 2-10 nTorr during epitaxy according to RGA measurements but it still could affect the layer and interfacial quality of $\mathrm{SiGe} / \mathrm{Si}$.

Many semiconductor manufacturers use in-situ high-resolution Xray diffractometer to examine the epi-layer quality. The instrument performs measurements of so-called rocking curves (RCs) when the incident angle is being scanned within an angle interval (usually around a symmetric reflection e.g. (004)) and the detector follows the diffracted beam to find out the layer and substrate peaks. The main criteria for RCs are the full-width-half-maximum (FWHM) of the layer peak and the number of interference fringes to find out the layer quality. In these measurements, a layer peak with small FWHM and many interference fringes are indications for a high quality of the epi-layer. In a rocking curve, the position of layer peak compared to the substrate peak determines the strain amount in the epi-layer. Figs. $4 \mathrm{a}$ and $4 \mathrm{~b}$ show a series of RCs from SiGe layers grown at different temperatures and oxygen exposure levels during the growth. These RCs indicate no change of SiGe layers FWHM nor the number of fringes.

Therefore, no defects could be detected for SiGe layers grown with oxygen partial pressure up to 160 nTorr. This value corresponds to an oxygen concentration of $\sim 1 \times 10^{19} \mathrm{~cm}^{-3}$ according to SIMS measurements. ${ }^{14}$ In fact, such a case may occur when a leakage incident occurs in the CVD reactor. The reason for such low resolution of $\mathrm{X}$-ray rocking curves in Figs. $4 \mathrm{a}$ and $4 \mathrm{~b}$ is due to the fact that these measurements are one-dimensional and the in-plane lattice parameter which is more sensitive to defects is not measured. Therefore, a two- dimensional analysis e.g. high-resolution reciprocal lattice mapping (HRRLM) is proposed as shown in Figs. 5a-5c. In all these maps, the integrity of the SiGe layers contours is preserved but a diffused scattering appears around the peak in Fig. 5b and Fig. 5c. Such diffused scattering is an indication of precipitates which cause a local disturbance in the SiGe matrix. ${ }^{18}$ The strain relaxation in these epi-layers was calculated from misfit parameters and it was below $1 \%$.

Oxygen contamination at $\mathrm{SiGe} / \mathrm{Si}$ interface forms oxide islands. ${ }^{25}$ Fig. 6 demonstrates the size of the formed oxide islands for all oxygen exposure experiments. A critical island size of $0.025 \mu \mathrm{m}^{2}$ for oxide

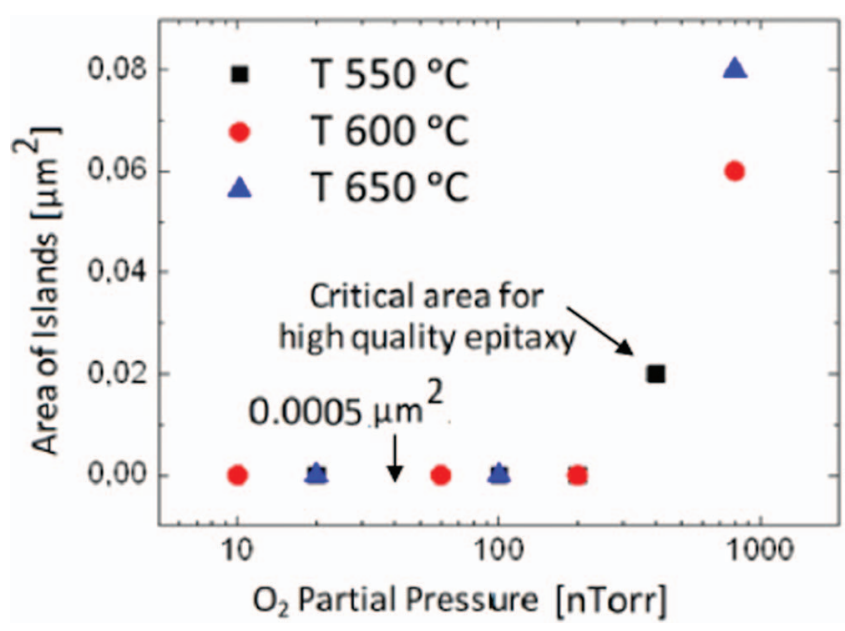

Figure 6. The area sizes of oxide islands formed at different growth temperatures and oxygen partial pressures. 


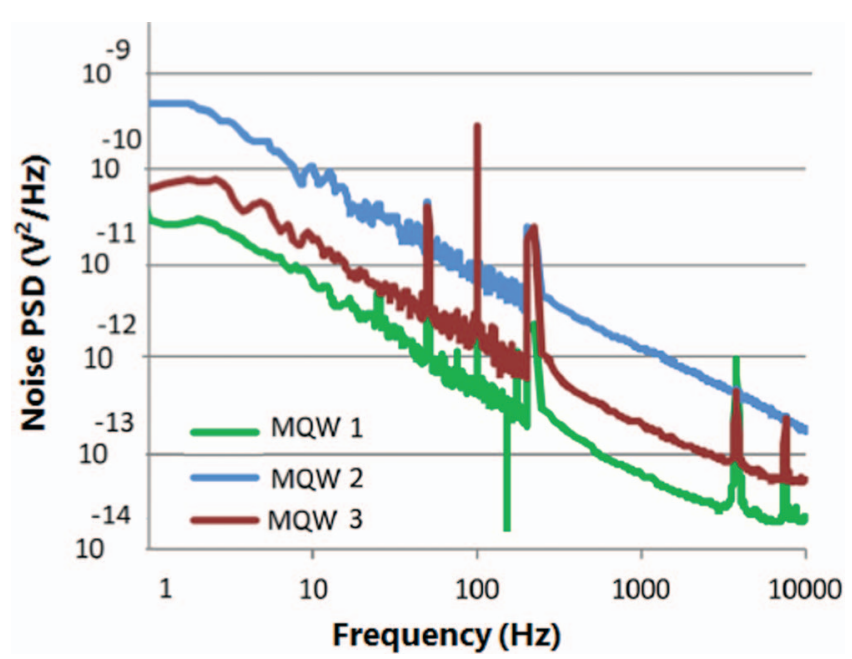

Figure 7. Power spectral density (PSD) curves from $\mathrm{Si}_{0.72} \mathrm{Ge}_{0.28}$ multilayers grown with 10 nTorr oxygen during epitaxy (in red color) and exposed at $\mathrm{SiGe} / \mathrm{Si}$ interface for $7 \mathrm{~min}$ prior to the growth (in light blue color). The reference is without oxygen contamination (in green color).

islands has to be reached in order to see any effect on epitaxy quality. This could be determined from HRRLMs when SiGe layer lattice parameters could be determined and the initial strain relaxation could be observed in presence of defects. For each sample, the size of oxide islands was obtained from SEM images. This means that the layer quality degradation could be distinguished by characterization tools when the size of defects is large and the defect density is high.

Furthermore, two sample structures were processed with a minor amount of oxygen contamination (10 nTorr) at $\mathrm{Si}_{0.78} \mathrm{Ge}_{0.28} / \mathrm{Si}$ interfaces (MQW2) or during the growth (MQW3). The background pressure of oxygen in the CVD reactor was measured to be 1 nTorr by RGA.

Fig. 7 shows the PSD curves of these samples and $\mathrm{K}_{1 / \mathrm{f}}$ values are summarized in Table I. In this figure, MQW1 is grown without oxygen contamination and is considered as reference sample.

In these samples, the reference sample indicates the lowest noise level $\left(\mathrm{K}_{1 / \mathrm{f}}=7 \times 10^{-11}\right)$. The noise level increases by a factor of 2 when the oxygen is within the grown layer $\left(\mathrm{K}_{1 / \mathrm{f}}=1.4 \times 10^{-10}\right)$ meanwhile the noise level increases by a factor of 10 when the oxygen is present at the interface $\left(\mathrm{K}_{1 / \mathrm{f}}=8 \times 10^{-10}\right)$.

This means that the transport of the carriers is very sensitive to the formed interfacial barriers (or defects) due to the oxygen islands formation. The first advice to the epitaxial growers is to avoid any long time interrupt during epitaxy (usually different purge steps are required) and to create growth recipes with fast purging.

The number of periods and the $\mathrm{Ge}$ content in $\mathrm{SiGe} / \mathrm{Si}$ are also two important integration issues for device application. Both of these factors determine the total strain energy in the multilayer structure which should not exceed the energy of relaxation. Noise measurement provides an indication of the increase in defect density by any change of periodicity and the Ge content.

As an example, $\mathrm{SiGe} / \mathrm{Si}$ multilayer structure is proposed as the active part for absorbing IR radiation in bolometers. For a high performance bolometer, a high temperature coefficient of resistance (TCR) and high SNR are two important criteria. Both of these criteria are dependent on the SiGe profile in the structure.

\section{Table I. Summary of oxygen exposure and $K_{1 / f}$.}

\begin{tabular}{llll} 
Sample description & MQW1 & MQW2 & MQW3 \\
\hline Oxygen exposure & No & Interfacial & Within SiGe layers \\
$\mathrm{K}_{1 / \mathrm{f}}$ for $25 \times 25 \mu \mathrm{m}^{2}$ & $7 \times 10^{-11}$ & $8 \times 10^{-10}$ & $1 \times 10^{-10}$
\end{tabular}

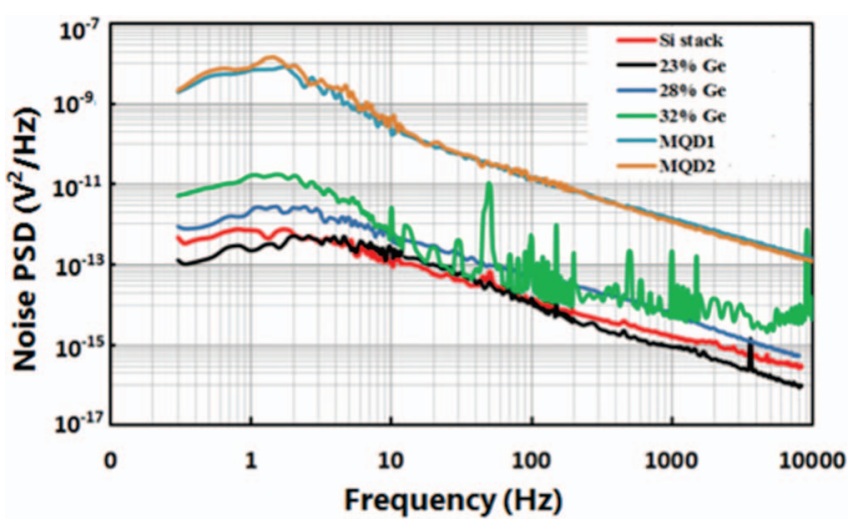

Figure 8. Power spectral density (PSD) curves from SiGe/Si multilayers (four periods) with Ge content of $21 \%, 28 \%$ and $32 \%$ and SiGe-dots with Ge content of $47 \%$ (MQD1) and $>47 \%$ (MQD2) multilayers (four periods). The reference sample is $\mathrm{Si}$ (in red color).

In this study, three samples with multilayers of $\mathrm{SiGe} / \mathrm{Si}$ were grown with Ge contents of $21 \%, 28 \%$ and $32 \%$ (MQW1, MQW2 and MQW3, respectively). Since the critical thickness of $\mathrm{SiGe}$ layers becomes impractically small for higher Ge contents, therefore $\mathrm{SiGe}$-dots/Si multilayers were instead grown. The composition (or strain amount) in the Ge dots depends on the size and the growth temperature. The latter parameter is also the main factor for the intermixing of $\mathrm{Si}$ into the Ge material. In this study, the SiGe dots are made by intermixing of $\mathrm{Si}$ and $\mathrm{Ge}$ dots at $600^{\circ} \mathrm{C}$. The X-ray analysis shows that the Ge content in MQD1 is $\sim 47 \%$. In order to reduce the intermixing, the growth temperature of Ge dots in MQD2 sample was decreased to $450^{\circ} \mathrm{C}$. Together with a Si reference sample, there are a total of six samples in this experiment.

Although, it is believed the Ge content in MQD2 is higher than MQD1 but X-ray results showed almost similar strain amount in these samples. This could be realized when the strain relaxation in MQD2 occurs due to high Ge content.

Fig. 8 shows the PSD curves versus applied frequency for all MQWs and MQDs samples and the extracted $\mathrm{K}_{1 / f}$ values are summarized in Table II. In this figure, the highest noise level is registered for MQD1 and MQD2 samples meanwhile this increased in MQWs by the increase in Ge content. Unexpectedly, the behavior of SNR of MQDs samples was very similar, despite their different Ge content. One plausible reason could be the amount of lattice distortion in these samples has been comparable. This could occur because of the strain relaxation in Ge dots with higher Ge content in MQD2 which makes the resulted strain amount in the same range as in MQD1. Further TEM investigations are required to confirm this theory.

A more detailed analysis was performed by cross-sectional HRSEM to evaluate the layer quality of samples as shown in Figs. 9a-9c. The analysis shows that MQW1 and MQW2 are deposited through 2D growth (see flat layers in MQW1) whereas MQW3 shows a Sinus-wave like growth similar to MQD1 which trends 3D growth (or uneven layer and the growth follows Stranski-krastanov mode in Figs. $9 \mathrm{~b}$ and $9 \mathrm{c}$ ).

The reason behind the unevenness of SiGe layers in MQW3 is because the layer thickness is close to the critical thickness. It is worth mentioning here that the strain relaxation of thin strained layers may occur through roughness (or unevenness) of the layers and not creating the misfit dislocation. ${ }^{27,28}$

In this case, one may conclude that the noise behavior is sensitive to the strain fluctuation within the layers. This could generate scatting of carriers during the transport through the multilayers. ${ }^{22}$

More analysis was performed to investigate the strain distribution in the SiGe dots. Figs. 10a-10c show HRRLMs and the rocking curves from a series of calibrating samples with $\mathrm{GeH}_{4}$ exposure times of 0.5 , 1, 2 and 4 minutes. The peaks from the Ge-dots in Figs. 10a and 10b are diffused but with a careful observation the maximum intensity 


\section{Table II. Summary of calculated $\mathrm{K}_{1 / \mathrm{f}}$ for SiGe/Si MQWs and MQDs.}

\begin{tabular}{|c|c|c|c|c|c|c|}
\hline Sample description & Ref. Si & MQW1 & MQW2 & MQW3 & MQD1 & MQD2 \\
\hline Ge content & 0 & 23 & 28 & 32 & 47 & $>47$ \\
\hline $\mathrm{K}_{1 / \mathrm{f}}$ for $25 \times 25 \mu \mathrm{m}^{2}$ & $9 \times 10^{-13}$ & $1 \times 10^{-12}$ & $3 \times 10^{-12}$ & $4.4 \times 10^{-12}$ & $2 \times 10^{-9}$ & $2 \times 10^{-9}$ \\
\hline
\end{tabular}

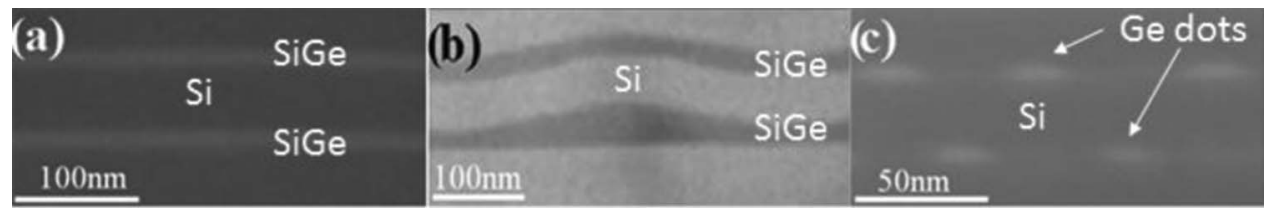

Figure 9. (a-c) Cross-section HRSEM micrographs from SiGe/Si multilayers with Ge contents of: (a) $28 \%$ and (b) $32 \%$ and (c) SiGe-dots with Ge content of 47\%. $\omega$ and $2 \theta$ stand for incident and diffracted angles, respectively.
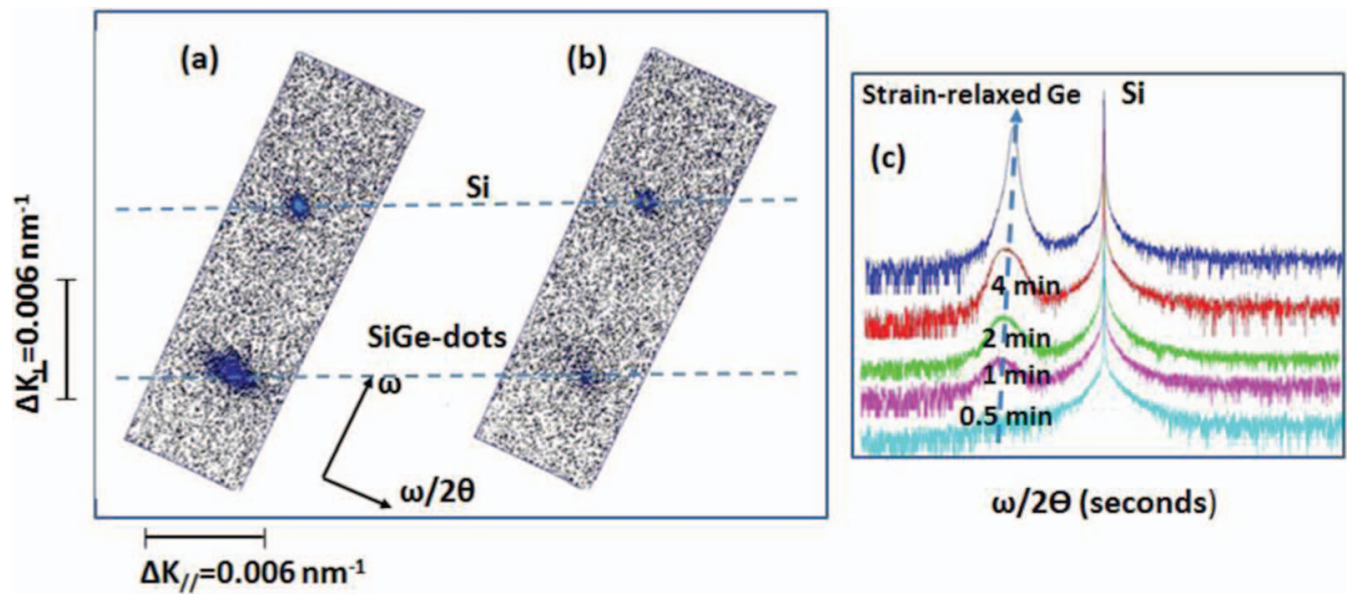

Figure 10. (a-c) HRRLMs from Ge (or SiGe) dots with $\mathrm{GeH}_{4}$ exposure times of: (a) 4 min and (b) 2 min and (c) $\mathrm{RC}$ of samples with $\mathrm{Ge}$ (or $\mathrm{SiGe)}$ with different exposure times. SR-Ge stands for strain relaxed layer with thickness of $300 \mathrm{~nm}$.

could be located and the relaxation amount was determined to be $80 \%$.

RCs in HRXRD are benefiting from very high X-ray intensity which is most necessary for these samples due to the small signal from Ge-dots. The RCs in Fig. 10c show the Ge peak shifts to the higher angles as the Ge-dots become larger. This is a sign of strain relaxation in Ge-dots. A full relaxation will occur when the dots in large scale coalesce (four minutes exposure). Therefore, it is most probable that a $\mathrm{GeH}_{4}$ exposure time shorter than one minute can provide the mostly strained dots (size of 3-10 nm).

\section{Conclusions}

SNR as a powerful tool to characterize engineered structures such as single crystalline dots or layers of $\mathrm{SiGe} / \mathrm{Si}$ in multilayer structures has been presented and the results were compared with structural and chemical material analysis. Low temperature epitaxy growth suffers from the quality of process gases and epitaxy environment in terms of oxygen or moisture level. Therefore, the $\mathrm{SiGe} / \mathrm{Si}$ interface or $\mathrm{SiGe}$ layer was examined by intentional exposure to oxygen in range of 2 1600 nTorr. No defects could be detected with oxygen partial pressures below $160 \mathrm{nTorr}$ either during or prior to $\mathrm{SiGe}$ growth. The calculated strain relaxation from the misfit parameters in these epi-layers was below $1 \%$. It was found that the transport of the carriers is very sensitive to the formed interfacial oxygen islands, thus, avoiding any long time interrupt during epitaxy or creating growth recipes with fast purging is suggested. By changing the Ge content in the structure, $\mathrm{K}_{1 / \mathrm{f}}$ was observed to be three orders of magnitude higher in MQDs (3D growth) compared to MQWs (2D growth) which is due to $80 \%$ relaxation amount in those multilayer structures. Ge-dots of larger size experience more relaxation which can be obtained from their Ge peak shifting to the higher angles. Best MQD sample was grown by $\mathrm{GeH}_{4}$ exposure time shorter than one minute which provided the mostly strained dots (size of 3-10 nm).

\section{References}

1. S. E. Thompson et al., IEEE Electron Device Lett., 25, 191 (2004).

2. K. Rim, J. Welser, J. L. Hoyt, and J. F. Gibbons, in International Electron Devices Meeting, p. 517 (1995).

3. G. L. Wang et al., J. Appl. Phys., 114, 123511 (2013).

4. J. Yun et al., IEEE Microw. Wirel. Components Lett., 25, 178 (2015).

5. R. Krithivasan et al., IEEE Electron Device Lett., 27, 567 (2006).

6. E. Onaran et al., Opt. Express, 20, 7608 (2012).

7. M. Kolahdouz, A. Afshar Farniya, M. Östling, and H. H. Radamson, Solid. State. Electron., 62, 72 (2011).

8. M. Kolahdouz, A. Afshar Farniya, L. Di Benedetto, and H. Radamson, Appl. Phys. Lett., 96, 213516 (2010).

9. H. H. Radamson, M. Kolahdouz, S. Shayestehaminzadeh, A. Afshar Farniya, and S. Wissmar, Appl. Phys. Lett., 97, 223507 (2010).

10. R. Soref, L. Friedman, and G. Sun, Superlattices Microstruct., 23, 427 (1996).

11. C.-H. Lai, K.-M. Chang, C.-F. Chen, and C.-T. Hsieh, Micro Nano Lett., 7, 729 (2012).

12. I. Saad et al., in IEEE International Conference on Semiconductor Electronics, p. 154 (2014).

13. Z. Kolahdouz et al., Mater. Sci. Eng. B, 177, 1542 (2012).

14. A. Abedin, M. Moeen, C. Cappetta, M. Östling, and H. H. Radamson, Thin Solid Films, In Press (2015).

15. M. Kolahdouz, A. Salemi, M. Moeen, M. Östling, and H. H. Radamson, J. Electrochem. Soc., 159, H478 (2012).

16. E. Van Kerschaver and G. Beaucarne, Prog. Photovoltaics Res. Appl., 14, 107 (2006)

17. E. Kasper, Properties of Strained and Relaxed Silicon Germanium, INSPEC, London, UK, (1995). 
18. H. Radamson and J. Hallstedt, J. Phys. Condens. Matter, 17, S2315 (2005).

19. J. Hallstedt, M. Blomqvist, P. O. A. Persson, L. Hultman, and H. H. Radamson, J. Appl. Phys., 95, 2397 (2004).

20. O. Nur et al., Appl. Phys. Lett., 64, 440 (1994).

21. F. N. Hooge, Phys. Lett. A, 29, 139 (1969)

22. M. Von Haartman and M. Östling, Low-frequency noise in advanced MOS devices, Springer Science \& Business Media, (2007).

23. R. Loo et al., in Solid State Phenomena, 145, 177, Trans Tech Publ (2009).
24. B. S. Meyerson, IBM J. Res. Dev, 34, 806 (1990).

25. A. Gruhle, G. Schmidt, H. Beneking, and H. Holzbrecher, J. Cryst. Growth, 110, 985 (1991)

26. F.-C. Hou, G. Bosman, E. Simoen, J. Vanhellemont, and C. Claeys, Electron Devices, IEEE Trans., 45, 2528 (1998).

27. M. Kolahdouz et al., IEEE Trans. Nanotechnol., 8, 291 (2009).

28. H. Radamson and L. Thylen, Monolithic Nanoscale Photonics-electronics Integration in Silicon and Other Group IV Elements, Academic Press (2014). 